\title{
Balanced Crystalloid Solution
}

National Cancer Institute

\section{Source}

National Cancer Institute. Balanced Crystalloid Solution. NCI Thesaurus. Code C126796.

A multiple electrolyte, isotonic, crystalloid solution for intravenous infusion containing sodium chloride, sodium gluconate, sodium acetate, potassium chloride and magnesium chloride, which can restore electrolyte balance, normalize $\mathrm{pH}$, and provide hydration. Upon intravenous administration, the balanced crystalloid solution will replace lost body fluids and electrolytes thereby providing hydration, normalizing electrolyte concentrations and regulating acid-base balance. 